\title{
Comparing and combining the Saskatoon, QMAP and COBE CMB maps
}

\author{
Yongzhong $\mathrm{Xu}^{1}$, Max Tegmark ${ }^{1}$, Angelica de Oliveira-Costa ${ }^{1}$, Mark J. Devlin ${ }^{1}$, Thomas Herbig ${ }^{2}$, Amber D. Miller ${ }^{3}$, \\ C. Barth Netterfield ${ }^{4}$, Lyman Page ${ }^{2}$ \\ ${ }^{1}$ Dept. of Physics, Univ. of Pennsylvania, Philadelphia, PA 19104; xuyz@physics.upenn.edu \\ ${ }^{2}$ Princeton University, Dept. of Physics, Princeton, NJ 08544 \\ ${ }^{3}$ Dept. of Astronomy and Astrophysics, Univ. of Chicago, Chicago, IL 60637 \\ ${ }^{4}$ Dept. of Physics and Astronomy, University of Toronto, Toronto, ON M5S1A7, Canada
}

(Submitted to Phys. Rev. D Oct. 27 2000, revised Dec. 22)

\begin{abstract}
We present a method for comparing and combining maps with different resolutions and beam shapes, and apply it to the Saskatoon, QMAP and COBE/DMR data sets. Although the Saskatoon and QMAP maps detect signal at the $21 \sigma$ and $40 \sigma$ levels, respectively, their difference is consistent with pure noise, placing strong limits on possible systematic errors. In particular, we obtain quantitative upper limits on relative calibration and pointing errors. Splitting the combined data by frequency shows similar consistency between the Ka- and Q-bands, placing limits on foreground contamination. The visual agreement between the maps is equally striking. Our combined QMAP+Saskatoon map, nicknamed QMASK, is publicly available at www.hep.upenn.edu/ xuyz/qmask.html together with its $6495 \times 6495$ noise covariance matrix. This thoroughly tested data set covers a large enough area (648 square degrees - currently the largest degree-scale map available) to allow a statistical comparison with COBE/DMR, showing good agreement.
\end{abstract}

98.62.Py, 98.65.Dx, 98.70.Vc, 98.80.Es

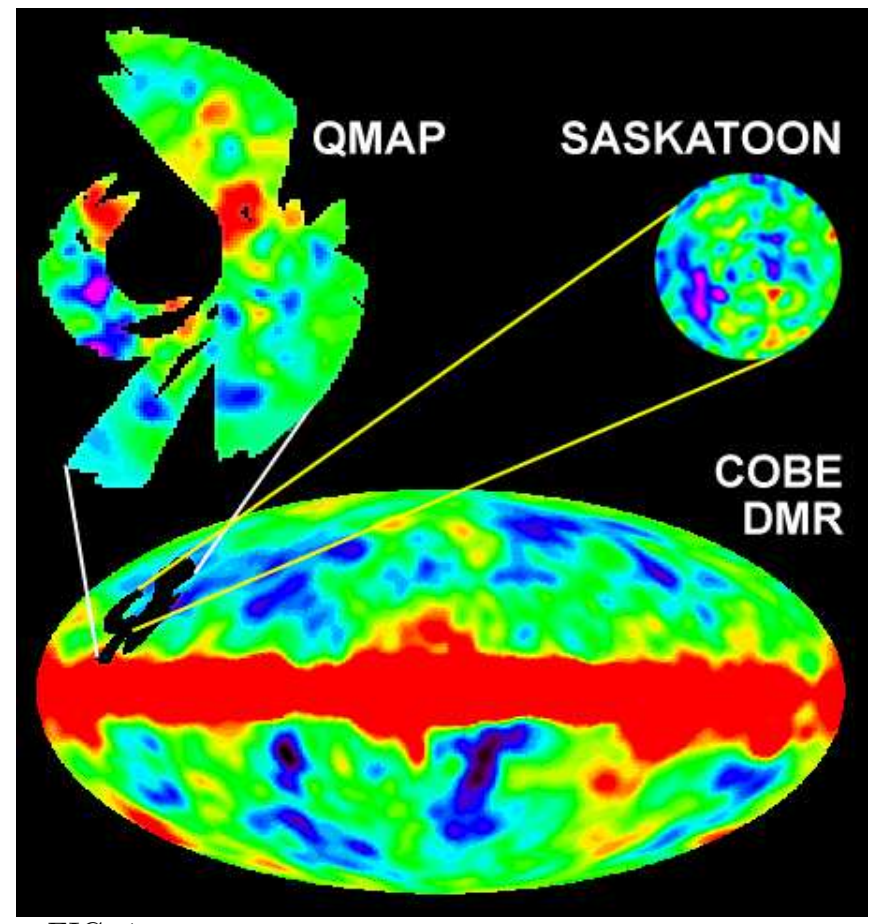

FIG. 1. The three maps that we will compare and combine are shown in Galactic coordinates. QMAP location in COBE map is shown in black.

\section{INTRODUCTION}

The cosmic microwave background (CMB) field is currently enjoying a bonanza of new high-quality data [1] 3 , which has triggered a surge of new papers about the implications for cosmological parameters [11. There is currently such wide interest in these cosmological results that it is tempting to temporarily ignore underlying assumptions. However, it is nonetheless important to consolidate these gains by careful study of the many technical analysis steps upon which they rest. This can be done at many levels. The experimental teams generally test for systematic errors at many steps in their data analysis pipeline, from data acquisition, cleaning, calibration and pointing reconstruction to mapmaking and power spectrum estimation. In addition, numerous detailed comparisons have been made between the angular power spectra $C_{\ell}$ measured by different experiments to determine whether they are all consistent [12 16,9]. However, such comparisons use only a very small fraction of the information at hand: average band powers, not the spatial phase information. A more powerful test (in the statistical sense of being more likely to discover systematic errors) involves a direct comparison of the sky maps from experiments that overlap in both spatial and angular coverage.

Such a comparison is straightforward for maps with identical resolution and beam shape, simply testing whether the difference map is consistent with pure detector noise. Such tests have been successfully performed for the COBE/DMR maps [17]. Unfortunately, comparisons are usually complicated by angular resolution differences between channels. Some experiments probe the sky in an even more complicated way, with, e.g., elliptical beams, double beams, triple beams, interferometric beams or complicated elongated software-modulated beams. Correlated noise further complicates the problem. Despite these difficulties, accurate comparisons between different experiments are crucial. Some of the best testimony to the quality of CMB maps comes from the success of such comparisons in the past - between FIRS and DMR [18], Tenerife and DMR [19], MSAM and Saskatoon [21,20], 
two years of Python data 22, three years of Saskatoon data [23], two flights of MSAM 24] and different channels of QMAP 25 27], Boomerang [2] and Maxima [3].

General methods have been developed for both comparing [20,28] and combining 28] arbitrarily complicated experiments. In this paper we will derive a technique for simplifying this task in practice, and apply it to compare and combine the three overlapping data sets shown in Figure if: COBE/DMR, Saskatoon and QMAP. Our motivation is threefold:

- To test and provide methods that can be used by experimental groups in the future.

- To search for systematic problems that may be relevant to ongoing and future experiments.

- To quality-test and publicly release the largest degree-scale map to date.

We stress that combining maps is not just a matter of making pretty pictures. Power spectra from different experiments are routinely combined as if their sample variance were independent. However, since this approximation breaks down whenever the underlying maps overlap in spatial and angular coverage, the only correct way to compute their combined power spectrum is to extract it from the combined map.

We present our methods in Section [1], present our results in Section III and summarize our conclusions in Section [V].

\section{METHOD}

We use the methods for combining and comparing maps presented in [28], which are most easily expressed with matrix notation. Given two data sets represented by the vectors $\mathbf{y}_{1}$ and $\mathbf{y}_{2}$, we write

$$
\mathbf{y}_{1}=\mathbf{A}_{1} \mathbf{x}+\mathbf{n}_{1}, \quad \mathbf{y}_{2}=\mathbf{A}_{2} \mathbf{x}+\mathbf{n}_{2}
$$

Here the vector $\mathbf{x}$ contains the temperature of the true sky at various locations (pixels). $\quad \mathbf{A}_{1}$ and $\mathbf{A}_{2}$ are two known matrices incorporating the pointing strategy and beam shape of each experiment. $\mathbf{n}_{\mathbf{1}}$ and $\mathbf{n}_{\mathbf{2}}$ are two random noise vectors with zero mean and known covariance matrices $\mathbf{N}_{1} \equiv\left\langle\mathbf{n}_{1} \mathbf{n}_{1}^{t}\right\rangle$ and $\mathbf{N}_{2} \equiv\left\langle\mathbf{n}_{2} \mathbf{n}_{2}^{t}\right\rangle$. It is convenient to define larger matrices and vectors

$$
\mathbf{A} \equiv\left(\begin{array}{c}
\mathbf{A}_{1} \\
\mathbf{A}_{2}
\end{array}\right), \quad \mathbf{y} \equiv\left(\begin{array}{l}
\mathbf{y}_{1} \\
\mathbf{y}_{2}
\end{array}\right), \quad \mathbf{n} \equiv\left(\begin{array}{c}
\mathbf{n}_{1} \\
\mathbf{n}_{2}
\end{array}\right)
$$

and to write the full noise covariance matrix as

$$
\mathbf{N} \equiv\left\langle\mathbf{n n}^{\mathbf{t}}\right\rangle=\left(\begin{array}{cc}
\mathbf{N}_{1} & \mathbf{N}_{12} \\
\mathbf{N}_{12}^{\mathrm{t}} & \mathbf{N}_{2}
\end{array}\right)
$$

We review the mathematical details of combining, filtering and comparing maps in Appendices A, B and $\mathrm{C}$, with some explicit details added (beyond [28]) that are useful when implementing these methods in practice.

We derive a new deconvolution method in Appendix D which substantially simplifies our calculations by eliminating the A-matrices above. In the generic case, deconvolution is strictly speaking impossible, since the matrix $\mathbf{A}$ is not invertible and certain pieces of information about $\mathbf{x}$ are simply not present in $\mathbf{y}$. It is common practice to find approximate solutions to such underdetermined problems using singular value decomposition or other techniques, but our goal is different: we need a deconvolved sky map $\tilde{\mathbf{x}}$ that can be analyzed as a true sky map with $\mathbf{A}=\mathbf{I}$ without approximations, shifting all complications into the new noise covariance matrix. The method derived in Appendix D is found to be stable numerically, and can be used both for "unsmoothing" low-resolution maps and to deconvolve more complicated oscillatory beam patterns such as that of the Saskatoon experiment.

\section{RESULTS}

In this section, we combine the QMAP, Saskatoon and COBE maps. We then perform a battery of tests for systematic errors by comparing the maps with each other, paying particular attention to possible calibration, pointing and foreground problems.

\section{A. Saskatoon Data}

The Saskatoon data set is very different from other data sets such as QMAP and COBE since it does not contain simple sky temperature measurements. Instead, the 2970 Saskatoon measurements are different linear combinations of the sky temperatures with rather complicated weight functions reminiscent of caterpillars - examples are plotted in 21,23. These measurements probe a circular sky patch with about $16^{\circ}$ diameter, centered on the the North Celestial Pole (NCP). In addition to the 2590 weight functions used in [23], which are all oriented like spokes of a wheel, we include the 380 "RING" data measurements, linear combinations in the perpendicular direction going around the periphery of the observing region.

We pixelize this sky region into 2016 pixels in the same coordinate system as QMAP, i.e., a simple square grid in gnomonic equal area projection, and define $\mathbf{x}$ to be the true sky convolved with a Gaussian beam of FWHM $0.68^{\circ}$. We compute the $2970 \times 2016$ matrix A using the software from the original Saskatoon analysis [31,21]. The rows of $\mathbf{A}$ have a vanishing sum since the beam functions are all insensitive to the monopole - they are normalized so that the absolute values sum to two. Using equations (D1) and (D2), we obtain a deconvolved Saskatoon CMB map $\tilde{\mathbf{x}}_{\mathrm{SASK}}$ and its corresponding covariance 
matrix $\boldsymbol{\Sigma}_{\text {SASK }}$. Figure 2 shows the Wiener-filtered Saskatoon map, which is visually almost identical to that in [23] except for the additional information from the RING data near the border.

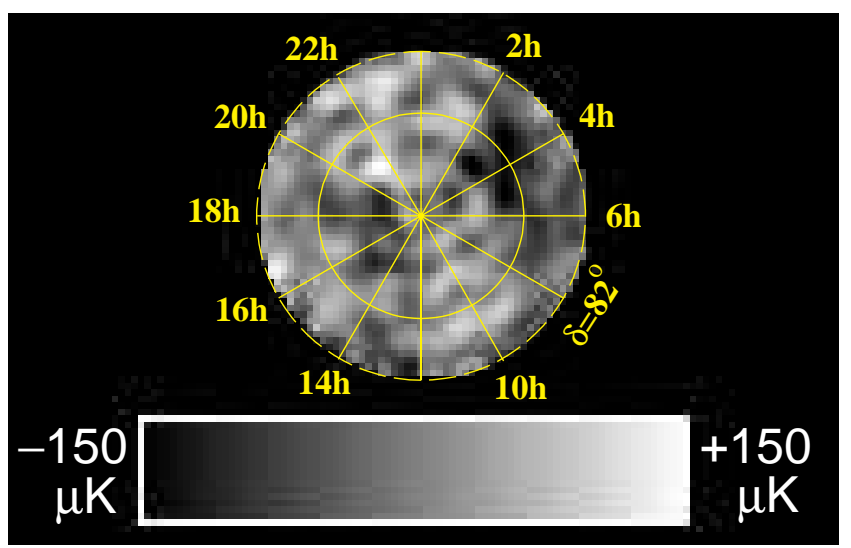

FIG. 2. Wiener-filtered Saskatoon map. The CMB temperature is shown in coordinates where the north celestial pole is at the center of a circle of $16^{\circ}$ diameter, with R.A. being zero at the top and increasing clockwise. In addition to the data included in the map of 23], "RING" data is included here. Note that the orientation of this and all following maps is different from that in Figure 1.

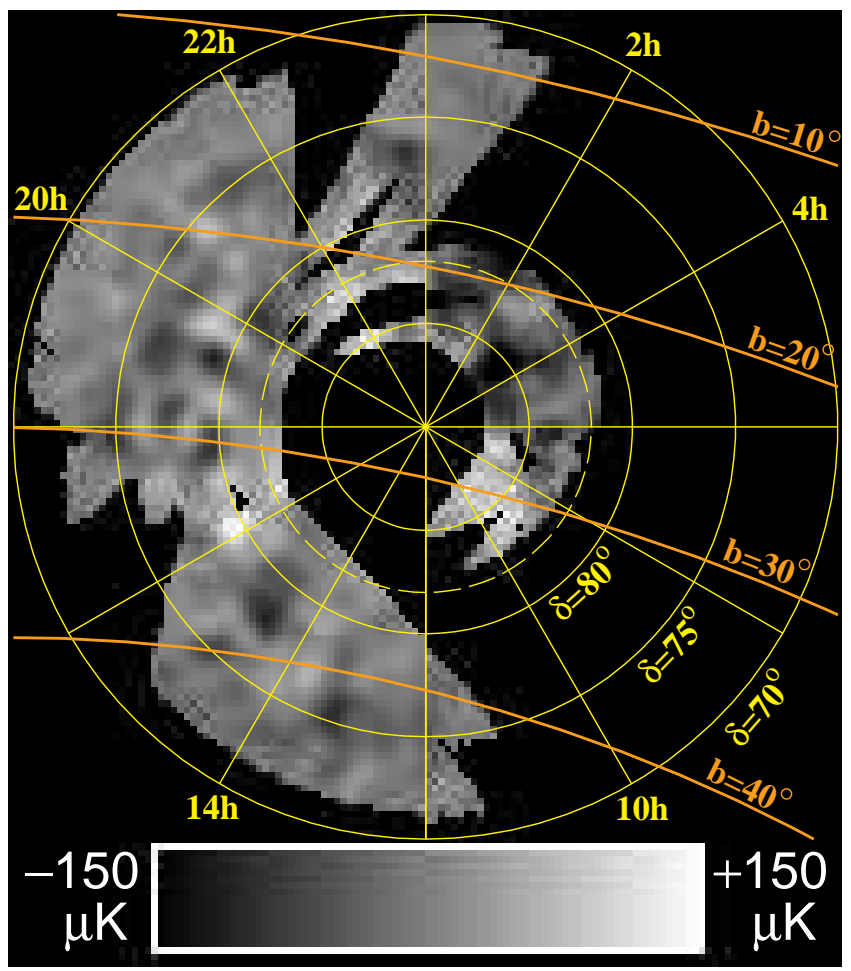

FIG. 3. Wiener-filtered QMAP map. The coordinates are the same as in the previous figure.

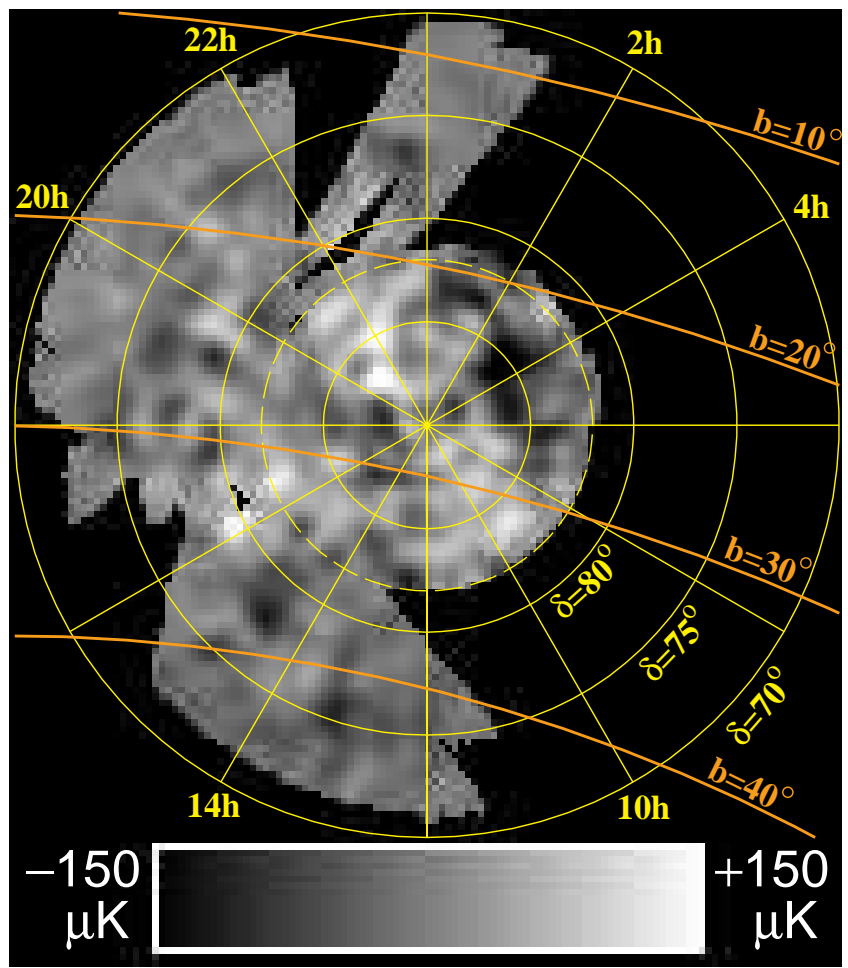

FIG. 4. Wiener-filtered map combining the QMAP and Saskatoon experiments. The coordinates are the same as in Figure 2

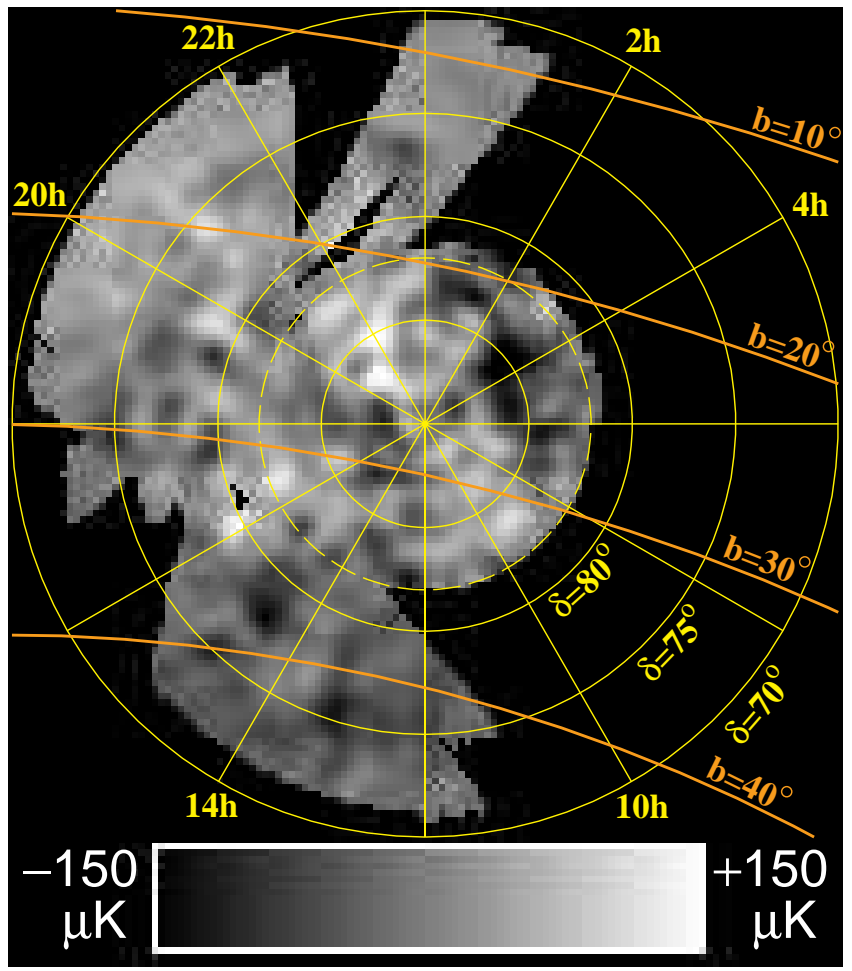

FIG. 5. Wiener-filtered map of the combined QMASK and COBE data. The coordinates are the same as in the previous figure. COBE adds only large-scale information. For example, the upper left region is brightened somewhat. 


\section{B. Combining QMAP with Saskatoon}

The QMAP data consists of Ka-band and Q-band measurements with angular resolution $0.89^{\circ}$ and $0.68^{\circ}$, respectively. We first deconvolve the Ka-band data to $0.68^{\circ}$ resolution using the method of Appendix $\mathrm{D}$. We then produce a unified QMAP data set $\left(\tilde{\mathbf{x}}_{\mathrm{QMAP}}, \boldsymbol{\Sigma}_{Q M A P}\right)$ with a single resolution, $0.68^{\circ}$, by combining the result with the Q-band as described in Appendix A, using equation (A11) to compute the final noise covariance $\boldsymbol{\Sigma}_{Q M A P}$ since the overlap between the two bands is only partial. This combined QMAP map has 5396 pixels covering a sky area of about 538 square degrees.

Combining QMAP and SASK is now a straightforward task, since the data sets $\tilde{\mathbf{x}}_{\mathrm{QMAP}}$ and $\tilde{\mathbf{x}}_{\mathrm{SASK}}$ have the same angular resolution and pixelization scheme. Since the spatial overlap is only partial, we once again use equation A11 to compute the combined noise covariance matrix. There are 917 overlapping pixels, so the combined map consists of 6495 pixels, covering a sky area of about 648 square degrees. We will nickname the combined data set "QMASK".

The main improvement in the combined is not the area covered (the QMASK map is only $20 \%$ larger than QMAP), but the signal-to-noise and the topology. SASK has excellent signal-to-noise in the region that it covers, which overlaps the most sensitive region of QMAP. Indeed, the two maps have comparable sensitivity in the overlap region, so both of them have substantial impact on the spatial features seen in Fig. 3. Filling in the "hole" in the map is also useful for comparing with lower resolution maps like $\mathrm{COBE}$ and for potential future applications, e.g., genus statistics, where a large contiguous area is desirable.

\section{Combining QMASK with COBE}

The COBE data 33, 34 has much lower angular resolution than QMASK (about 7.08 ), and the pixel size of COBE is much bigger than that of QMASK as well (about $2.6^{\circ} \times 2.6^{\circ}$ ). In total, there are 6144 pixels in the whole COBE sky map. We select those pixels whose centers are within the QMASK map and at least $3^{\circ}$ away from the perimeter. Only $58 \mathrm{COBE}$ pixels satisfy these criteria.

We first deconvolve this COBE data to the QMASK angular resolution, using the method of Appendix D with A 1 being a $58 \times 6495$ matrix with a Gaussian COBE beam on each row, normalized to sum to unity. As input, we use the inverse-variance weighted average of the 53 and $90 \mathrm{GHz}$ COBE/DMR channels [17]. By construction, our resulting COBE and QMASK maps overlap each other perfectly, so we obtain our combined map by simply using equations A5 and A6.

At first glance, Figure 4 and Figure 5 look very similar. However, inspecting them more carefully reveals that al- though the small scale patterns are the same, the upper part in Figure 5 is brighter than that in Figure 4 (this is related to the QMASK cold spot that we will discuss subsection III E). In other words, since COBE contains only large scale information, this is precisely what it has added in Figure 5, leaving the small scale structure unaffected.

\section{Comparing QMAP with Saskatoon}

As mentioned above, there are 917 pixels overlapping between the QMAP and Saskatoon data sets. After extracting these pixels and their two noise covariance matrices from the full maps, we can compare the two experiments using the null-buster test described in Appendix $\emptyset$.
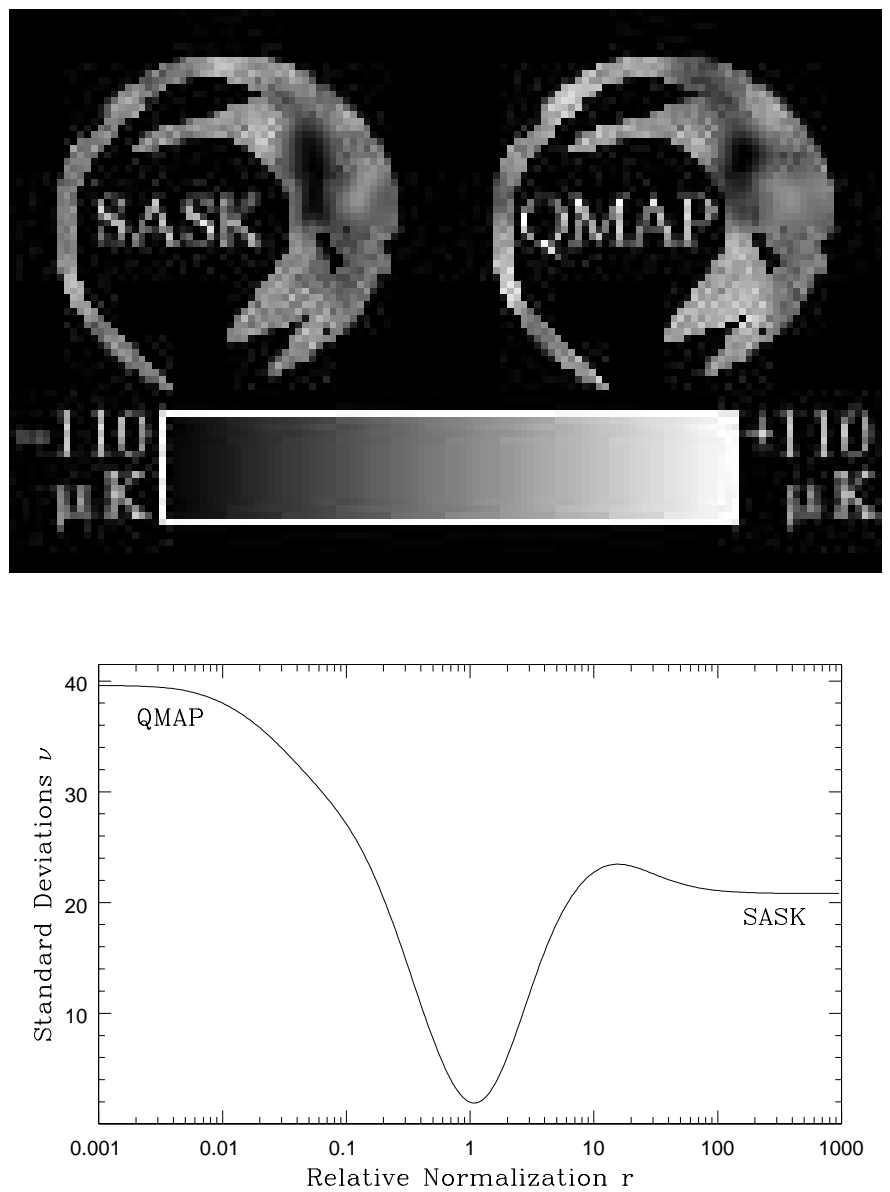

FIG. 6. Comparison of QMAP (left) and Saskatoon (right). The upper panel shows both maps Wiener filtered with the same weighting in the overlap region. The lower panel shows the number of standard deviations ("sigmas") at which the difference map $\tilde{\mathbf{x}}_{\mathrm{QMAP}}-r \tilde{\mathbf{x}}_{\mathrm{SASK}}$ is inconsistent with mere noise. Note that this is only for the overlap region. 


\section{Visual comparison}

Before delving into statistical details, it is useful to compare the two maps visually. Comparing plots of the raw maps $\tilde{\mathbf{x}}$ is rather useless, since they are so noisy. Unfortunately, comparing plots of the two Wiener-filtered maps like figures 25 is not ideal either: since the noise matrices $\mathbf{N}_{1}$ and $\mathbf{N}_{2}$ are different, this would entail comparing apples and oranges, since the two Wiener-filtered maps would be smoothed and weighted differently. For instance, if a prominent spot in one map is invisible in the other, this could either signal a problem or be due to that particular region being very noisy in the second map and therefore suppressed by the Wiener filtering.

To circumvent this problem, we Wiener-filter both maps exactly in the same way,

$$
\mathbf{x}_{i}^{w}=\mathbf{S}\left[\mathbf{S}+\mathbf{N}_{1}+\mathbf{N}_{2}\right]^{-1} \tilde{\mathbf{x}}_{i}, \quad i=1,2 .
$$

In other words, we replace the individual noise covariance matrices by their sum, so that the map will only show information that is accurately measured by both experiments. These maps $\mathbf{x}_{1}^{w}$ and $\mathbf{x}_{2}^{w}$ are compared in the upper part of Figure 6, and are seen to look encouragingly similar.

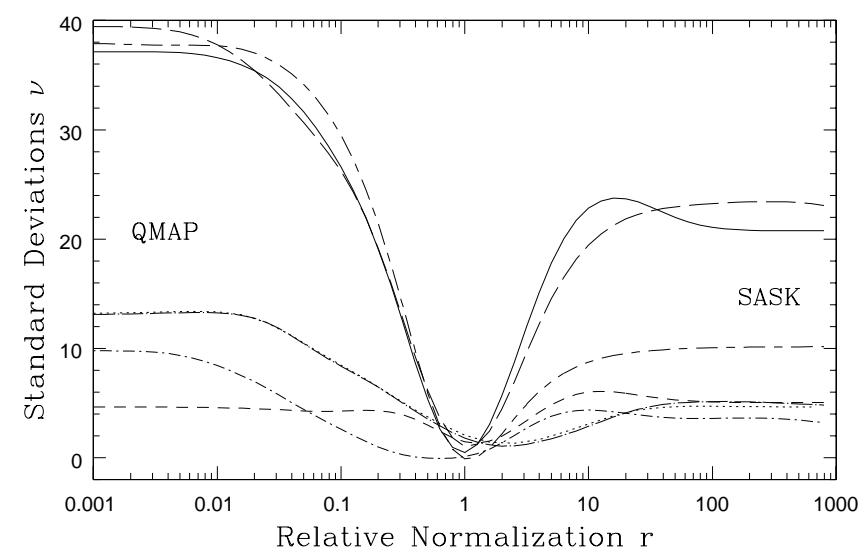

FIG. 7. Same as previous figure, but comparing Saskatoon with various subsets of the QMAP data. The curves correspond to Ka-band (dotted), Q-band (solid), flight 1 (short-dashed), flight 2 (long-dashed), f2Ka12(dot-long-dashed), f2Q12 (dot-short-dashed) and f2Q34 (short-dashed-long dashed).

\section{Tests for systematic and calibration errors}

The lower plot in Figure 6 shows the results of applying the null-buster test to the difference map $\tilde{\mathbf{x}}_{\mathrm{QMAP}}-$ $r \tilde{\mathbf{x}}_{\text {SASK }}$ for different values of the constant $r$. (The corresponding noise covariance matrix $\mathbf{N}=\mathbf{N}_{1}+\mathbf{r}^{2} \mathbf{N}_{2}$.) The left part of the curve where $r \ll 1$ is dominated by information from QMAP, and we see that QMAP alone (the $r=0$ case) is inconsistent with noise at about the $40 \sigma$ level. Similarly, we see that the Saskatoon map alone (the case $\mathbf{r}=\infty$ ) is inconsistent with noise at about the $20 \sigma$ level. Note that these significance levels are still higher for the full maps - here we are limiting ourselves to the sky region where they overlap.

In summary, both the QMAP and Saskatoon maps contain plenty of signal. Is this signal consistent between the two maps? The answer is given by the most interesting point on the curve, where the relative normalization value $r=1$. In the absence of systematic or calibration errors, the corresponding difference map should contain pure noise. In our case, when $r=1$, the difference map $\tilde{\mathbf{x}}_{\mathrm{QMAP}}-\tilde{\mathbf{x}}_{\mathrm{SASK}}$ is seen to be consistent with pure noise, i.e., less than $2 \sigma$ away from zero. The strong signal seen in both maps therefore appears to be a true sky signal, with no evidence for significant systematic errors in either QMAP or Saskatoon.

The QMAP experiment consists of two flights, each with two frequencies (Ka and Q-band) and three slightly different observing regions. We label these six sub-maps f1Ka12, f1Q2 and f1Q34 (from flight 1) and f2Ka12, f2Q12 and f2Q34 (from flight 2). The pointing and calibration analyses for these two flights were completely separate, and the map-making algorithm was applied separately for these six sub-maps 25 27. To investigate possible problems with these individual sub-maps that may have been averaged away in the combined analysis, we repeat the comparison with Saskatoon separately for each one. The results are shown in Figure 7. None of these curves show any evidence for systematic or calibration errors. The f2Q34 map is seen to contain the strongest signal, inconsistent with noise at the $38 \sigma$ level. This is because f2Q34 contains a striking cold spot — we will return to this in more detail in subsection III G.

Table 1. Summary of map comparisons. The first three lines give the number of "sigmas" at which map 1, the difference map and map 2, respectively, are inconsistent with noise. The remaining lines give the best fit value and limits on the relative calibration $r$, or, for the Ka vs. Q case, the spectral index $\beta$.

\begin{tabular}{|c|c|c|c|}
\hline & QMAP vs SK & QMASK vs COBE & Ka vs Q band \\
\hline$\nu(r=0)$ & 40 & 62 & 40 \\
$\nu(r=1)$ & 1.97 & -0.64 & -0.53 \\
$\nu(r=\infty)$ & 21 & 3 & 26 \\
\hline$r_{\min }(3 \sigma)$ & 0.79 & 0.09 & $-2.73^{*}$ \\
$r_{\min }(2 \sigma)$ & 0.95 & 0.13 & $-2.2^{*}$ \\
$r_{\min }(1 \sigma)$ & & 0.2 & $-1.7^{*}$ \\
$r_{\text {best }}$ & 1.1 & 1.25 & $0 .^{*}$ \\
$r_{\max }(1 \sigma)$ & & 11.7 & $1.42^{*}$ \\
$r_{\max }(2 \sigma)$ & 1.2 & 20 & $1.9^{*}$ \\
$r_{\max }(3 \sigma)$ & 1.48 & 63 & $2.2^{*}$ \\
\hline
\end{tabular}

* values of the power spectral index $\beta$ in subsection IIIF

The null-buster curves are interesting at more than just the points $r=0,1$, and $\infty$; the entire region near $r=1$ places limits on calibration errors. A relative calibration 
error of say $10 \%$ would shift the minimum of the curve sideways to 0.9 or 1.1 , depending on whether the QMAP or Saskatoon map was too high. We can therefore place limits on calibration errors by reading off the $r$-values where noise is ruled out at say 2 sigma. For instance, the QMAP-Saskatoon comparison constrains the relative calibration error to be less than $20 \%(3 \sigma)$, and the $r$ values for different significance levels are shown in table 1. This method may prove quite useful for upcoming experiments that have higher sensitivity.

\section{Pointing tests}

Our comparison method can also be used to test for relative pointing errors, as a complement to the standard lower-level pointing tests that are routinely made using point sources etc. Although an overall sideways shift of a single map will not affect the measured power spectrum, such errors can become disastrous if the map is combined with another one.

As an illustration of such a test, we compare the f2Q34 map with the Saskatoon map with the null-buster test (setting $r=1$ ) after shifting it vertically and horizontally by an integer number of pixels. Figure 8 shows that there is no evidence for pointing error although we cannot give a strong constraint. Just as the calibration test, this pointing test based on $\mathrm{CMB}$ maps alone is likely to be useful for upcoming high-sensitivity experiments.

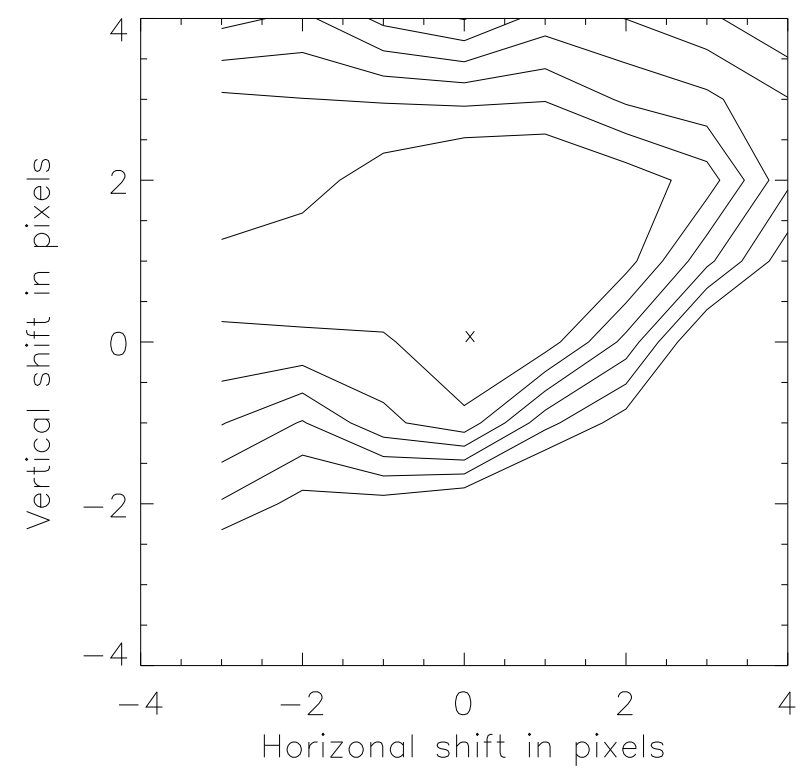

FIG. 8. Test of the relative pointing of QMAP and Saskatoon. The curves show the number of "sigmas" at which the difference map is inconsistent with noise when the QMAP map is shifted vertically and horizontally. Starting from the inside, the contours are at $1,2,3,4,5$, and $6 \sigma$ respectively. Cross indicates no shift. The pixels are squares of side $0.3125^{\circ}$.
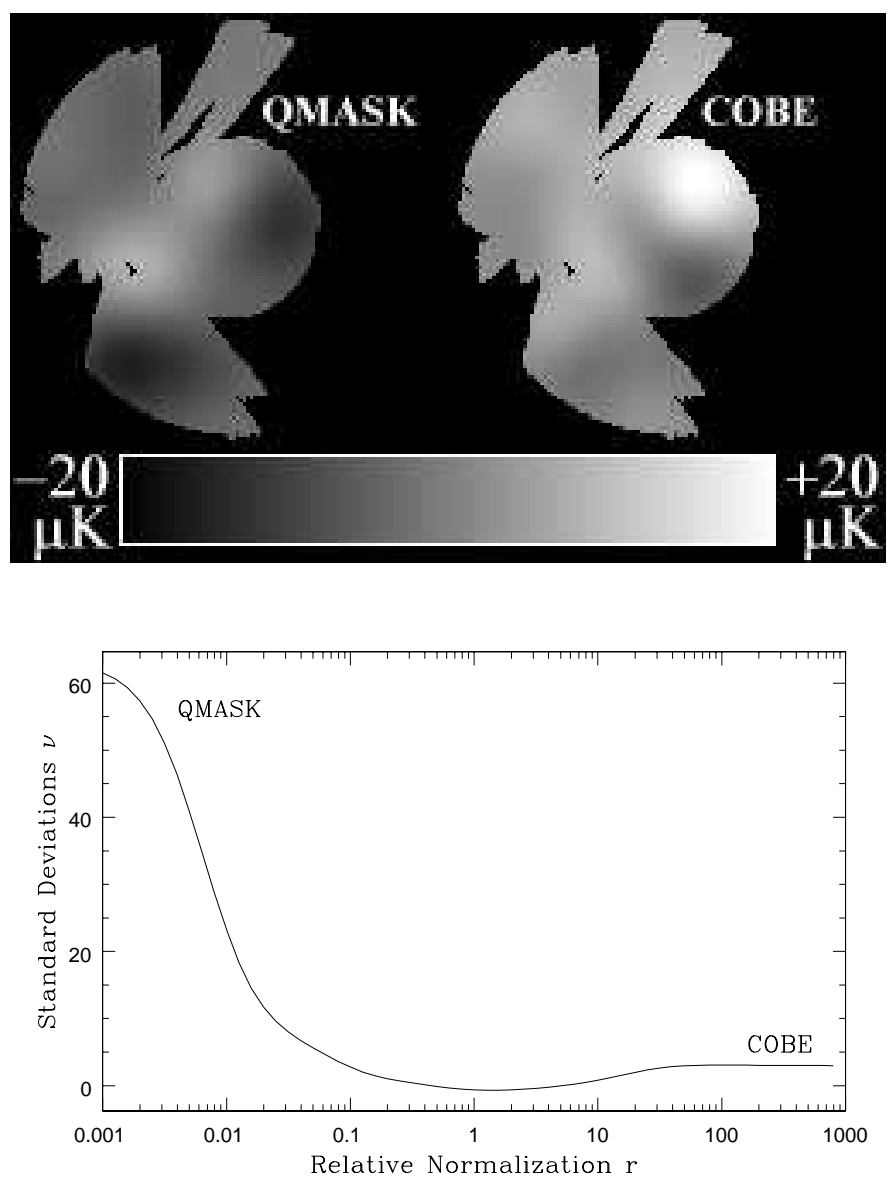

FIG. 9. Comparison of QMASK (left) with COBE (right). The upper panel shows both maps Wiener filtered with the same weighting in the overlap region, using equation (4). The lower panel shows the number of standard deviations ("sigmas") at which the difference map $\tilde{\mathbf{x}}_{\mathrm{QMASK}}-r \tilde{\mathbf{x}}_{\mathrm{COBE}}$ is inconsistent with mere noise, and illustrates that the visual discrepancy at " 2 o'clock" is consistent with a fluctuation in the (correlated) noise.

\section{E. Comparing QMASK with COBE}

Our QMASK map and our deconvolved COBE map cover the exact same sky region, so there are 6495 pixels in the overlap maps. Generating Figure 9, which compares these two maps, therefore involved a marathon computer run, processing the $6495 \times 6495$ matrices of equation (C1) for each $r$-value. f $^{-}$As can be seen in the lower panel of Figure 9, the QMASK data is inconsistent

* If CPU time had been an issue, this calculation could have been accelerated by binning the QMASK pixels into larger ones. This would give essentially the same answer, since the null-buster test gives statistical weight only to modes where both maps are sensitive - in this case, to large scale modes only. 
with noise at about the $62 \sigma$-level, whereas the COBE data is inconsistent with noise slightly above the $3 \sigma$ level. Since neither the QMAP nor the Saskatoon experiments were designed to probe such large angular scales, it is quite encouraging that the QMASK-COBE difference map $(r=1)$ is seen to be consistent with pure noise. Since the minimum of the curve is so broad, however, we obtain no interesting constraints on calibration errors.

The upper panel in Figure 9 shows that the two maps look fairly similar considering the weak $(3 \sigma)$ COBE signal, with the notable exception of the upper right part of the Saskatoon disk. Here COBE shows a hot spot whereas QMASK shows a cold spot. We will return to this issue in more detail below, in subsection III G. When generating these two maps, we used the same equalweighting Wiener filtering method that was described in subsubsection III D 1. This is particularly important here, since the QMASK and COBE have such dramatically different angular resolutions - in contrast, a visual comparison of the Wiener filtered COBE map with the normal Wiener filtered QMASK map from Figure 14 is rather useless, since the latter is dominated by smallscale fluctuations.

\section{F. Foreground constraints}

The previous two sections used map comparisons to test for calibration and pointing errors. Here we will compare maps at different frequencies to constrain the spectrum of the detected sky signal.

The presence of foreground contamination (see 35 for a recent review) has been quantified for both the Saskatoon [32 and QMAP [36] experiments by crosscorrelating the maps with various foreground templates. The dominant foreground emission is expected to be due to synchrotron radiation, free-free emission and (vibrational and spinning) dust emission, from both the Milky Way (seen as diffuse emission) and other galaxies (seen as point sources). These cross-correlation analyses concluded that foregrounds played only a subdominant role in Saskatoon and QMAP.

By comparing the Ka- and Q-band maps, we are able to place a direct constraint on the frequency dependence of the signal. Both Saskatoon and QMAP observe in both of these frequency bands. We therefore repeat the analysis described above (Saskatoon deconvolution, merging with QMAP, etc.) separately for each of the two bands. The upper panel of Figure 10 shows equalweighting Wiener-filtered maps for Ka-band and Q-band in the sky region that was observed at both frequencies, showing that they visually agree well.

If we fit the frequency dependence by a power law $\delta T(\nu) \propto \nu^{\beta}$ over the narrow frequency range in question $\left(\nu_{\mathrm{Ka}} \approx 30 \mathrm{GHz}, \nu_{\mathrm{Ka}} \approx 40 \mathrm{GHz}\right)$, then characteristic spectral indices are $\beta \sim-2.8$ for synchrotron, $\beta \sim-2.15$ for free-free emission, $\beta \sim-3$ for spinning dust and $\beta \sim 2$ for vibrating dust. By definition, $\beta=0$ for CMB. If the sky signal in our maps obeyed $\delta T(\nu) \propto \nu^{\beta}$, then the difference map $\tilde{\mathbf{x}}_{\mathrm{Ka}}-r \tilde{\mathbf{x}}_{\mathrm{Q}}$ would contain pure noise when $r$ was such that

$$
\beta=\frac{\lg r}{\lg \left(\nu_{\mathrm{Ka}} / \nu_{\mathrm{Q}}\right)} .
$$

The lower panel of Figure 10 is therefore plotted with $\beta$ rather than $r$ on the horizontal axis. Insisting that the difference map not be inconsistent with noise at more than $1 \sigma$ gives the spectral index constraint $\beta=0.0_{-1.7}^{+1.4}$, which is inconsistent with the signal being any single one of the foregrounds mentioned above.
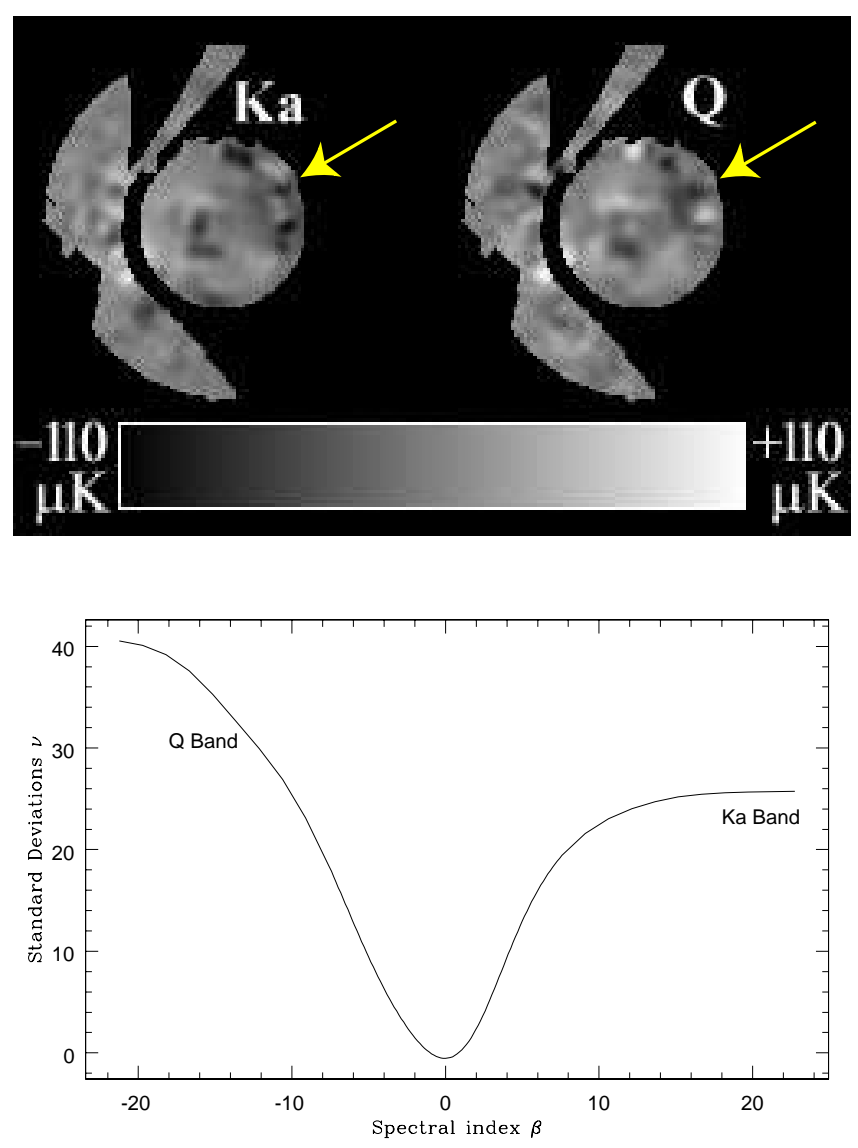

FIG. 10. Comparison of QMASK at two different frequencies, Ka-band (left) and Q-band (right). The upper panel shows both maps Wiener filtered with the same weighting in the overlap region that was observed at both frequencies. Arrows indicate the coldest spot discussed below. The lower panel shows the number of standard deviations ("sigmas") at which the difference map $\tilde{\mathbf{x}}_{\mathrm{Ka}}-r \tilde{\mathbf{x}}_{\mathrm{Q}}$ is inconsistent with mere noise.

\section{G. The coldest spot}

Up until now, we have presented a battery of tests for systematic errors and other problems, all of which passed. 
However, our maps did turn up one somewhat anomalous feature: an unusually cold spot around "two o'clock" in the Saskatoon disk. The spot's coldest pixel in the QMASK map is located at $\mathrm{RA}=3^{h} 20^{m}, \mathrm{DEC}=84^{\circ} 55^{\prime}$. Here the Wiener-filtered $Q$-band map in Figure 10 gives $\delta T \approx-230 \mu \mathrm{K}$. For comparison, the expected rms fluctuations in this map are $27 \mu \mathrm{K}$ from detector noise and $49 \mu \mathrm{K}$ from CMB fluctuations (for the "concordance" power spectrum of [1]), summing to $56 \mu \mathrm{K}$ in quadrature. Taken at face value, this would indicate that the spot is a $-4.1 \sigma$ fluctuation. For completeness, this section describes a number of additional tests performed in an attempt to clarify its nature.

It is unlikely that the cold spot is due entirely to systematic error, since it is clearly detected by both QMAP (covered by the Q3 and Q4 detectors from in flight 2) and Saskatoon. The Saskatoon experiment even detected this spot independently in each of its three observing seasons 21,23. Indeed, the reason that the f2Q34 map shows the most spectacular agreement with Saskatoon in Figure 7 is that $\mathrm{f} 2 \mathrm{Q} 34$ is the only QMAP map that covers the area containing this spot.

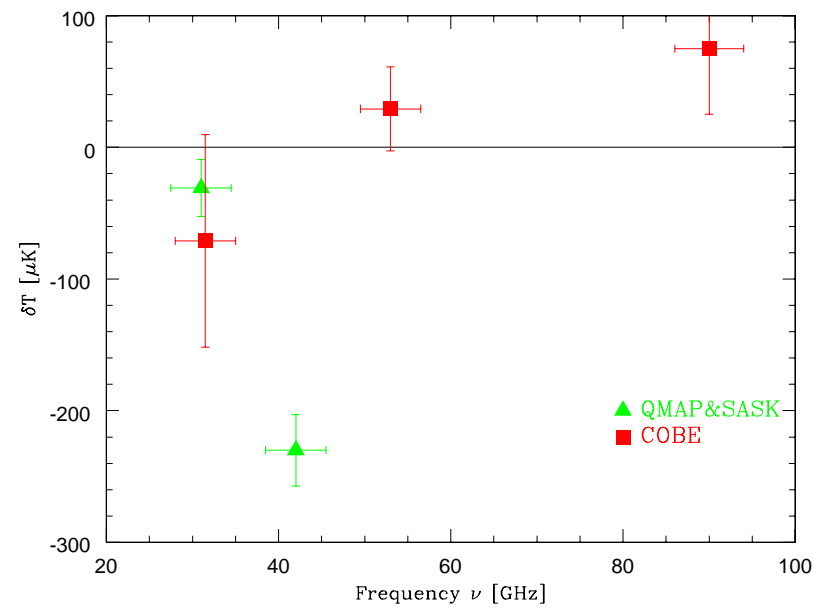

FIG. 11. The temperature towards $\mathrm{RA}=3^{h} 20^{m}, \mathrm{DEC}=84^{\circ} 55^{\prime}$ at different frequencies. Errors bars correspond to detector noise alone. Note that these points cannot be interpreted as a spectrum of this sky region, since the COBE points have much lower resolution and the two QMASK points have been Wiener filtered with different weights, pushing them closer to zero than the underlying sky temperature.

We have plotted all available microwave measurements of this region in Figure 11 as a function of frequency, including both the Ka- and Q-band measurements from QMASK and the COBE/DMR observations at 31.5, 53 and $90 \mathrm{GHz}$ 17. Unfortunately, Figure 11 is not an actual spectrum of the spot. It is more of a comparison of apples and oranges, since the measurements differ dramatically in angular resolution ( $7^{\circ}$ for COBE) and the QMASK maps are Wiener-filtered. Since Wiener filtering always pushes the signal towards zero when noise is present, the QMASK points should be interpreted as lower limits on $|\delta T|$ — the true sky temperature is likely to be even colder.

On the seven degree scale probed by COBE, no evidence is seen for a cold spot (this can also be seen in the map of Figure 9), and the COBE spectrum of this region appears consistent with $\mathrm{CMB}$, i.e., $\beta=0$. One possible interpretation is that a small cold region resolved by QMAP and Saskatoon is partly smoothed away by COBE.

Unfortunately, this sky patch is not covered by QMAP in Ka-band, so the Ka-information comes from Saskatoon alone and is therefore noisier than the Q-band measurement. This means that the Wiener-filtering has suppressed the Ka-band more, as well as lowered its resolution by more aggressive smoothing. However, the expected rms CMB fluctuations in the Wiener-filtered maps are not nearly as different as the data points in Figure $11(-31 \mu \mathrm{K}$ at Ka-band and $-230 \mu \mathrm{K}$ at Q-band), indicating that the low Q-band temperature does not persist fully down to Ka-band. This argues against both a CMB origin and a thermal SZ-origin, which would cause a cold spot that was essentially frequency-independent for $\nu<100 \mathrm{GHz}$.

All other known microwave foregrounds produce hot rather than cold spots. Some sort of absorption process also appears unlikely, since the absorbing medium would have to be colder than $3 \mathrm{~K}$. No relevant foreground emission or X-ray cluster is found in radio, infrared or X-ray maps of the region. Some adjacent dust emission is seen in the IRAS $100 \mu \mathrm{m}$ map [32], which could potentially make this region look cold in contrast (since none of our maps are sensitive to the monopole mode and measure merely relative temperatures), but only at the level of about $10 \mu \mathrm{K}$.

In Q-band, the spot is observed by QMAP only in flight two and only in the Q3 and Q4 channels. The latter dominates statistically, and has a $13 \%$ calibration uncertainty. However, Saskatoon also observes the spot in Q-band, and both experiments measure around $-200 \mu \mathrm{K}$ in their individual Wiener filtered maps.

The explanation is fairly likely to be something mundane, since a 4-sigma fluctuation (which should happen about once for every 16,000 independent pixels) is not extremely unlikely when many pixels are considered - if the effective number of independent regions in the Wiener filtered map is $10^{2}$ taking the smoothing into account, the significance level gets downgraded to $(1-1 / 16000)^{100} \approx 99 \%$ 37.

In summary, the cold spot is definitely out there at some level, but we have no single simple interpretation of what is causing it. Its unusual spectrum argues against a non-Gaussian CMB fluctuation, foreground contamination and an SZ signal. The most likely remaining explanation is a confluence of a less extreme CMB cold spot, noise fluctuations, calibration uncertainty and perhaps some small systematic error. We have described this spot in such detail simply to ensure that no hints of problems with the data get swept under the rug. 
The MAP satellite should resolve this puzzle next year, observing the spot with high sensitivity and resolution at 22, 30, 40, 60 and $90 \mathrm{GHz}$.

\section{DISCUSSION}

We have presented methods for comparing and combining CMB maps and applied them to the QMAP, Saskatoon and COBE DMR data sets. We found that these methods were able to place interesting constraints on calibration and pointing problems, foreground contaminations and systematic errors in general. This should make them quite useful for ongoing and upcoming high-precision experiments.

The data sets passed our entire battery of consistency tests, placing strong limits on systematic errors. Although the Saskatoon and QMAP maps detect signal at the $21 \sigma$ and $40 \sigma$ levels in the overlap region, respectively, their difference is consistent with pure noise. Our combined QMAP + Saskatoon map, nicknamed QMASK, covers a large enough area to allow a statistical comparison with COBE/DMR, showing good agreement.

The one surprise that our battery of tests turned up is that a small region around $\left(\mathrm{RA}=3^{h} 20^{m}, \mathrm{DEC}=84^{\circ} 55^{\prime}\right)$ appears unusually cold, mainly in Q-band. Its unusual frequency dependence argues against non-Gaussian CMB fluctuations, SZ-signal and known foregrounds.

The QMASK map presented here has been made publicly available at www.hep.upenn.edu/ xuyz/qmask.html together with its $6495 \times 6495$ noise covariance matrix. With its 648 square degrees, this thoroughly tested data set is currently the largest degree-scale map available, detecting signal at a level exceeding $60 \sigma$.

Support for this work was provided by NSF grants AST00-71213 and NSF 9732960, NASA grant NAG59194 (né NAG5-6034), a NASA GSRP grant, the University of Pennsylvania Research Foundation, the Zaccheus Daniel Foundation and the Dodds Foundation. The QMAP and SASK experiments were supported by NSF grants PH 89-21378, 92-22952, 96-00015, NYI (to LP); NASA grants NAGW-1482, NAGW-2801, NAG56034; Hubble Fellowships HF-01044.01-93A (to TH) and HF-01084.01-96A (to MT); a Cottrell Award from the Research Corporation (to LP) and a David and Lucile Packard Foundation Fellowship (to LP). The COBE data sets were developed by the NASA Goddard Space Flight Center under the guidance of the COBE Science Working Group and were provided by the NSSDC.

\section{APPENDIX A: COMBINING MAPS}

The combined map $\tilde{\mathbf{x}}$ defined by

$$
\tilde{\mathbf{x}} \equiv\left[\mathbf{A}^{t} \mathbf{N}^{-1} \mathbf{A}\right]^{-1} \mathbf{A}^{t} \mathbf{N}^{-1} \mathbf{y}
$$

can be shown to be unbiased $(\langle\tilde{\mathbf{x}}\rangle=\mathbf{x})$, to minimize the rms noise in each pixel and, if the noise properties are Gaussian, to retain all information about the true sky $\mathbf{x}$ that was present in the two original maps [28]. The corresponding covariance matrix of the noise $\epsilon \equiv \tilde{\mathbf{x}}-\mathbf{x}$ is

$$
\boldsymbol{\Sigma} \equiv\left\langle\varepsilon \varepsilon^{t}\right\rangle=\left[\mathbf{A}^{t} \mathbf{N}^{-1} \mathbf{A}\right]^{-1}
$$

In all cases treated in this paper, the noise is uncorrelated between different maps $\left(\left(\mathbf{N}_{12}=0\right)\right.$, which simplifies these equations to

$$
\begin{aligned}
& \tilde{\mathbf{x}}=\boldsymbol{\Sigma}\left[\mathbf{A}_{1}^{t} \mathbf{N}_{1}^{-1} \mathbf{y}_{1}+\mathbf{A}_{2}^{t} \mathbf{N}_{2}^{-1} \mathbf{y}_{2}\right] \\
& \boldsymbol{\Sigma}=\left[\mathbf{A}_{1}^{t} \mathbf{N}_{1}^{-1} \mathbf{A}_{1}+\mathbf{A}_{2}^{t} \mathbf{N}_{2}^{-1} \mathbf{A}_{2}\right]^{-1}
\end{aligned}
$$

Thanks to the deconvolution technique that will be described in Appendix D, we will generally face the much simpler case where the two data sets are two sky maps with the exact same angular resolutions, i.e., the case $\mathbf{A}_{1}=\mathbf{A}_{2}=\mathbf{I}$, reducing the last two equations to simply

$$
\begin{aligned}
& \tilde{\mathbf{x}}=\boldsymbol{\Sigma}\left[\mathbf{N}_{1}^{-1} \mathbf{y}_{1}+\mathbf{N}_{2}^{-1} \mathbf{y}_{2}\right], \\
& \boldsymbol{\Sigma}=\left[\mathbf{N}_{1}^{-1}+\mathbf{N}_{2}^{-1}\right]^{-1} .
\end{aligned}
$$

For the case of only a single pixel, we recognize this as a familiar inverse-variance weighting. More generally, if the two noise matrices can be simultaneously diagonalized, we see that this combination scheme corresponds to an inverse-variance weighting eigenmode by eigenmode.

Generally maps overlap only partially, so we need only apply this matrix method in the common region. Yet care needs to be taken in computing the noise covariance matrix $\boldsymbol{\Sigma}$ of the final map, since it will contain correlations between the common region and the rest. Let us split the noise vectors for the two maps as

$$
\mathbf{n}_{1}=\left(\begin{array}{c}
\mathbf{n}_{a} \\
\mathbf{n}_{c 1}
\end{array}\right), \quad \mathbf{n}_{2}=\left(\begin{array}{c}
\mathbf{n}_{b} \\
\mathbf{n}_{c 2}
\end{array}\right)
$$

where the subscript $c$ refers to the common region of the two maps whereas $a$ and $b$ refer to the regions that only belong to maps 1 and 2 , respectively. We write the corresponding covariance matrices as

$$
\begin{aligned}
& \mathbf{N}_{1} \equiv\left\langle\mathbf{n}_{1} \mathbf{n}_{1}^{t}\right\rangle=\left(\begin{array}{cc}
\mathbf{N}_{a} & \left\langle\mathbf{n}_{a} \mathbf{n}_{c 1}^{t}\right\rangle \\
\left\langle\mathbf{n}_{c 1} \mathbf{n}_{a}^{t}\right\rangle & \mathbf{N}_{c 1}
\end{array}\right), \\
& \mathbf{N}_{2} \equiv\left\langle\mathbf{n}_{2} \mathbf{n}_{2}^{t}\right\rangle=\left(\begin{array}{cc}
\mathbf{N}_{b} & \left\langle\mathbf{n}_{b} \mathbf{n}_{c 2}^{t}\right\rangle \\
\left\langle\mathbf{n}_{c 2} \mathbf{n}_{b}^{t}\right\rangle & \mathbf{N}_{c 2}
\end{array}\right),
\end{aligned}
$$

Substituting equation (11) into equation (A3), we obtain the noise vector $\mathbf{n}_{c}$ for the combined map in the common region:

$$
\mathbf{n}_{c}=\boldsymbol{\Sigma}_{c}\left[\mathbf{A}_{c 1}^{t} \mathbf{N}_{c 1}^{-1} \mathbf{n}_{c 1}+\mathbf{A}_{c 2}^{t} \mathbf{N}_{c 2}^{-1} \mathbf{n}_{c 2}\right]
$$

where $\boldsymbol{\Sigma}_{c}$ is given by equation (A4) for the common part. The final combined noise covariance matrix $\boldsymbol{\Sigma}$ for the noise vector $\left(\mathbf{n}_{a}, \mathbf{n}_{b}, \mathbf{n}_{c}\right)$ of the combined map is therefore 


$$
\boldsymbol{\Sigma}=\left(\begin{array}{ccc}
\mathbf{N}_{a} & \mathbf{0} & \left\langle\mathbf{n}_{a} \mathbf{n}_{c}^{t}\right\rangle \\
\mathbf{0} & \mathbf{N}_{b} & \left\langle\mathbf{n}_{b} \mathbf{n}_{c}^{t}\right\rangle \\
\left\langle\left[\mathbf{n}_{a}^{o} \mathbf{n}_{c}^{t}\right]^{t}\right\rangle & \left\langle\left[\mathbf{n}_{b} \mathbf{n}_{c}^{t}\right]^{t}\right\rangle & \boldsymbol{\Sigma}_{c}
\end{array}\right)
$$

where

$$
\begin{aligned}
\left\langle\mathbf{n}_{a} \mathbf{n}_{c}^{t}\right\rangle & =\left\langle\mathbf{n}_{a} \mathbf{n}_{c 1}^{t}\right\rangle \mathbf{N}_{c 1}^{-1} \mathbf{A}_{c 1} \boldsymbol{\Sigma}_{c} \\
\left\langle\mathbf{n}_{b} \mathbf{n}_{c}^{t}\right\rangle & =\left\langle\mathbf{n}_{b} \mathbf{n}_{c 2}^{t}\right\rangle \mathbf{N}_{c 2}^{-1} \mathbf{A}_{c 2} \boldsymbol{\Sigma}_{c} .
\end{aligned}
$$

We need to use these expressions repeatedly in this paper to combine partially overlapping maps, e.g., combining the different QMAP flights with each other and combining QMAP with Saskatoon.

\section{APPENDIX B: PLOTTING MAPS}

Although the map $\tilde{\mathbf{x}}$ contains all the sky information from an experiment, plotting it is not very useful when some modes are much more noisy than others, thereby dominating the visual image. For this reason, it has become standard in the community to plot the corresponding Wiener filtered map, defined as

$$
\mathbf{x}_{w} \equiv \mathbf{S}[\mathbf{S}+\mathbf{N}]^{-1} \tilde{\mathbf{x}}
$$

where $\mathbf{S}$ is an estimate of the covariance matrix due to sky signal. Throughout this paper, we use the $\mathbf{S}$-matrix corresponding to the "concordance" power spectrum from [11], which agrees well with all current CMB measurements.

\section{APPENDIX C: COMPARING MAPS}

Here we discuss the issue of how to test whether two data sets are consistent or display evidence of systematic errors. Specifically, is there some true sky $\mathbf{x}$ such that the data sets $\mathbf{y}_{1}$ and $\mathbf{y}_{2}$ are consistent with equation (1)? Let us first consider the simplest case where the two data sets sample the sky in the same way, that is, $\mathbf{A}_{1}=\mathbf{A}_{2}$. Consider two hypotheses:

$H_{0}$ : The null hypothesis $H_{0}$ that there are no systematic errors, so that the difference map $z \equiv \mathbf{y}_{1}-\mathbf{y}_{2}$ consists of pure noise with zero mean and covariance matrix $\left\langle\mathbf{z z}^{t}\right\rangle=\mathbf{N} \equiv \mathbf{N}_{1}+\mathbf{N}_{2}$.

$H_{1}$ : The alternative hypothesis that the difference map $\mathbf{z}$ consists of some signal besides noise, i.e., $\langle\mathbf{z}\rangle=0$, and $\left\langle\mathbf{z z}^{t}\right\rangle=\mathbf{N}+\mathbf{S}$ for some signal covariance matrix S.

The "null-buster" statistic 28]

$$
\nu \equiv \frac{\mathbf{z}^{t} \mathbf{N}^{-1} \mathbf{S} \mathbf{N}^{-1} \mathbf{z}-\operatorname{tr}\left\{\mathbf{N}^{-1} \mathbf{S}\right\}}{\left[2 \operatorname{tr}\left\{\mathbf{N}^{-1} \mathbf{S} \mathbf{N}^{-1} \mathbf{S}\right\}\right]^{1 / 2}}
$$

can be shown to rule out the null hypothesis $H_{0}$ with the largest average significance $\langle\nu\rangle$ if $H_{1}$ is true, and can be interpreted as the number of "sigmas" at which $H_{0}$ is ruled out [28]. Note that for the special case $\mathbf{S} \propto \mathbf{N}$, it reduces to simply $\nu=\left(\chi^{2}-n\right) / \sqrt{2 n}$, where $\chi^{2} \equiv \mathbf{z}^{t} \mathbf{N}^{-1} \mathbf{z}$ is a standard chi-squared statistic. The null-buster test can therefore be viewed as a generalized $\chi^{2}$-test which places more weight on those particular modes where the expected signal-to-noise is high. It has proven successful comparing both microwave background maps 25, 27] and galaxy distribution [29,30].

To evaluate equation (C1) in practice, it is useful to Cholesky decompose the noise matrix as $\mathbf{N}=\mathbf{L L}^{t}$ and compute the matrix $\mathbf{R} \equiv \mathbf{L}^{-1} \mathbf{S L}^{-t}$. The remainder of the calculation now becomes trivial, since $\operatorname{tr}\left\{\mathbf{N}^{-1} \mathbf{S}\right\}=$ $\operatorname{tr} \mathbf{R}=\sum \mathbf{R}_{i i}$ and $\operatorname{tr}\left\{\mathbf{N}^{-1} \mathbf{S} \mathbf{N}^{-1} \mathbf{S}\right\}=\operatorname{tr} \mathbf{R}^{2}=\sum\left(\mathbf{R}_{i j}\right)^{2}$.

For the general case when $\mathbf{A}_{1} \neq \mathbf{A}_{2}$, the situation is more complicated, since it is non-trivial to construct a difference map which is free of sky signal. A technique involving signal-to-noise eigenmode analysis has been derived for this case [28], but it is unfortunately rather complicated and cumbersome to implement. Below we present a simpler method that eliminates need for this by reducing the general problem to the simple case $\mathbf{A}_{1}=\mathbf{A}_{2}=\mathbf{I}$.

\section{APPENDIX D: DECONVOLVING MAPS}

In this section, we present a method for inverting equation (11), i.e., for undoing the convolution with beam and scanning effects given by the $\mathbf{A}$-matrices.

\section{a. Why is it useful?}

As we will see, this simplifies calculations by eliminating all A-matrices, encoding the corresponding complications and correlations in the noise covariance matrices.

When comparing or combining two maps, it is generally undesirable to smooth the higher resolution one down to the lower resolution of the other, since this destroys information. Moreover, this tends to cause numerical instabilities by making the smoothed noise covariance matrix poorly conditioned. We will see that, surprisingly, deconvolution can be better conditioned than convolution/smoothing.

This deconvolution (elimination of A-matrices) is useful not only for comparing data sets as mentioned above, but for combining them as well. The complication stems from the fact that the sky is sampled by only a finite number of pixels, so to avoid problems with undersampling, $\mathbf{x}$ must be the "true sky" beam-smoothed map with some finite angular resolution. If we do not deconvolve, but use equations (A3) and (A4) to combine two data sets with different angular resolutions, say $\theta_{1}=0.89^{\circ}$ and $\theta_{2}=0.68^{\circ}$ for the QMAP Ka- and Q-band maps, respectively, it is natural to define $\mathbf{x}$ to have the higher 
$\left(0.68^{\circ}\right)$ resolution, setting $\mathbf{A}_{2}=\mathbf{I}$ and letting $\mathbf{A}_{1}$ incorporate the extra smoothing $\Delta \theta=\sqrt{\theta_{1}^{2}-\theta_{2}^{2}}$ in the lower resolution map. The resulting map will now contain two kinds of pixels: ones with resolution $\theta_{2}$ in the region covered only by map 2 and with the higher resolution $\theta_{1}$ elsewhere. If we need to combine this with a third map, the relevant smoothing scale unfortunately becomes undefined near the boundary between the two resolutions. Deconvolution eliminates all these problems.

\section{b. How does it work?}

In the generic case, deconvolution is strictly speaking impossible, since the matrix $\mathbf{A}$ is not invertible f and certain pieces of information about $\mathbf{x}$ are simply not present in $\mathbf{y}$. It is common practice to find approximate solutions to such under-determined problems using singular value decomposition or other techniques, but our goal here is different. We wish to compute a vector $\tilde{\mathbf{x}}$ that can be analyzed as a true sky map. Specifically, we want analysis of $(\tilde{\mathbf{x}}, \boldsymbol{\Sigma})$ to give exactly the same results as analysis of $(\mathbf{y}, \mathbf{N}, \mathbf{A})$ for all cosmological applications, say Wiener filtering or power spectrum estimation.

Our basic idea is to accept that certain modes in the map $\tilde{\mathbf{x}}$ cannot be recovered, and to record this information in the noise covariance matrix $\boldsymbol{\Sigma}$ by assigning a huge variance to these modes. Any subsequent analysis (say Wiener filtering or power spectrum estimation) will then automatically assign essentially zero weight to these modes.

In practice, we find it conceptually useful to imagine combining our data $\mathbf{y}_{1}$ with a "virtual map" $\mathbf{y}_{2}$ that is so noisy that it contains essentially no information, yet has the angular resolution $\theta$ that we wish to deconvolve down to, i.e., $\mathbf{A}_{2}=\mathbf{I}$. Although, as we will see, this virtual map never enters the calculations in practice, it is a useful notion for intuitively understanding what the deconvolution technique does. Specifically, let us take the noise in the virtual map to be uncorrelated, with noise covariance matrix $\mathbf{N}_{2}=\sigma^{2} \mathbf{I}$ for some very large noise level $\sigma$. Equations ( $\mathrm{A} 3$ ) and (A4) give

$$
\begin{aligned}
\tilde{\mathbf{x}} & =\boldsymbol{\Sigma} \mathbf{A}_{1}^{t} \mathbf{N}_{1}^{-1} \mathbf{y}_{1}+\sigma^{-2} \boldsymbol{\Sigma} \mathbf{y}_{2}, \\
\boldsymbol{\Sigma} & =\left[\mathbf{A}_{1}^{t} \mathbf{N}_{1}^{-1} \mathbf{A}_{1}+\sigma^{-2} \mathbf{I}\right]^{-1} .
\end{aligned}
$$

In the limit $\sigma \mapsto \infty, \tilde{\mathbf{x}}$ will clearly become independent of the virtual temperature map $\mathbf{y}_{2}$ except for the "junk modes" which have infinite variance according to $\boldsymbol{\Sigma}$. For convenience, we therefore set $\mathbf{y}_{2}=\mathbf{0}$ in practice.

\footnotetext{
${ }^{\dagger}$ Specifically, the problem is that $\mathbf{A}$ generically has a nonzero null space, i.e., that there are non-zero vectors $\mathbf{x}$ such that $\mathbf{A x}=\mathbf{0}$.

${ }^{\ddagger}$ An alternative approach would be to set $\mathbf{y}_{2}$ equal to a
}

This deconvolution method has exactly the property we want as long as $\sigma$ is orders of magnitude larger than the pixel signal due to CMB. If we were to choose $\sigma$ to be too small, then the virtual map would contribute a nonnegligible amount of information and bias the results. If we were to choose $\sigma$ to be too large, however, the matrix $\boldsymbol{\Sigma}$ would contain some enormous eigenvalues (since $\mathbf{A}_{1}^{t} \mathbf{N}_{1} \mathbf{A}_{1}$ is typically not invertible) and be poorly conditioned, which could cause numerical problems in subsequent analysis. We performed a series of numerical tests to assess these problems, and found that with $n \lesssim 10^{4}$ pixels and double precision arithmetic, $\sigma=10^{4} \mu \mathrm{K}$ was a good compromise that produced neither of these two problems. We will therefore use this choice throughout the present paper.

$$
\text { c. Tests }
$$

As a first test of the method, we deconvolve (or "unsmooth") a map $\mathbf{y}_{1}$ with resolution $\theta_{1}$ into a map with resolution $\theta_{2}\left(\theta_{2}<\theta_{1}\right)$. For this case,

$$
\left(\mathbf{A}_{1}\right)_{i j}=\frac{1}{2 \pi \Delta \theta^{2}} e^{-\frac{\theta_{i j}^{2}}{2 \Delta \theta^{2}}}
$$

where $\theta_{i j}=\cos ^{-1}\left(\widehat{\mathbf{r}}_{i} \cdot \widehat{\mathbf{r}}_{j}\right)$ is the angular separation between pixels $i$ and $j$, and $\Delta \theta \equiv \sqrt{\theta_{1}^{2}-\theta_{2}^{2}}$ is the extra smoothing to be undone. Specifically, we unsmooth the QMAP Ka-band data to obtain the same resolution as the Q-band data has, from $0.89^{\circ}$ to $0.68^{\circ}$. We then Wiener filtered both the original and unsmoothed versions of the map, obtaining virtually identical results.

As a second test, we deconvolve the Saskatoon data $\mathbf{y}$ into a map $\tilde{\mathbf{x}}$ using the full (and rather complicated) A-matrix described in [23]. We then Wiener-filter $\tilde{\mathbf{x}}$ and obtain a map virtually identical to the one that was computed in [23] - the latter was computed with a completely different method which circumvented the map step altogether. The details of these maps have already been presented above.

Both of these tests thus confirm what we expect theoretically: that the deconvolved map $(\tilde{\mathbf{x}}, \boldsymbol{\Sigma})$ contains exactly the same information about the true sky as the input data $(\mathbf{y}, \mathbf{N}, \mathbf{A})$, no more and no less.

Monte-Carlo generated map of independent Gaussian random variables with standard deviation $\sigma$ - although this results in different numerical values in $\tilde{\mathbf{x}}$, it will of course not change the results of any subsequent cosmological analysis of $\tilde{\mathbf{x}}$, since only the "junk modes" are different. 
[1] E. Gawiser and J. Silk, Phys. Rept. 333-334, 245-267 (2000).

[2] P. de Bernardis et al., Nature 404, 955 (2000).

[3] S. Hanany et al., ApJL 545, L5 (2000).

[4] A. E. Lange et al., astro-ph/0005004 (2000).

[5] M. Tegmark and M. Zaldarriaga, Phys. Rev. Lett. 85, 2240 (2000).

[6] A. Balbi et al., ApJL 545, L1 (2000).

[7] S. L. Bridle et al., astro-ph/0006170 (2000).

[8] W. Hu, M. Fukugita, M. Zaldarriaga, and M. Tegmark astro-ph/0006436; $A p J$, in press.

[9] A. Jaffe et al., astro-ph/0007333 (2000).

[10] W. Kinney, A. Melchiorri, and A. Riotto, astroph/0007375 (2000).

[11] M. Tegmark, M. Zaldarriaga, and A. J. S Hamilton, Phys. Rev. D 63, 043007 (2001).

[12] D. Scott, J. Silk, and M. White, Science 268, 829 (1995).

[13] C. H. Lineweaver, ApJL 505, L69 (1998).

[14] M. Tegmark, ApJL 514, L69 (1999).

[15] S. Dodelson and L. Knox, Phys. Rev. Lett 84, 3523 (2000).

[16] M. Tegmark and M. Zaldarriaga, ApJ 544, 30T (2000).

[17] C. L. Bennett et al., ApJ 464, L1 (1996).

[18] K. Ganga, E. Cheng, S. Meyer, and L. Page, ApJL 410, L57 (1993).

[19] C. H. Lineweaver et al., ApJ 448, 482 (1995).

[20] L. Knox, J. R. Bond, A. H. Jaffe, M. Segal, and D. Charbonneau, astro-ph/9803272 (1998).

[21] C. B. Netterfield, M. J. Devlin, N. Jarosik, L. A. Page, and E. J. Wollack; ApJ, 474, 47 (1997)

[22] J. E. Ruhl et al., ApJL 453, L1 (1995).

[23] M. Tegmark et al., ApJL 474, L77 (1996a).

[24] C. A. Inman et al., ApJL 478, L1 (1997).

[25] M. Devlin, A. de Oliveira-Costa, T. Herbig, A. D. Miller, C. B. Netterfield, L. A. Page, and M. Tegmark, ApJL 509, L77 (1998).

[26] T. Herbig et al., ApJL 509, L73 (1998).

[27] A. de Oliveira-Costa, M. Devlin, T. Herbig, A. D. Miller, C. B. Netterfield, L. A. Page, and M. Tegmark, ApJL 509, L77 (1998).

[28] M. Tegmark, ApJ 519, 513 (1999).

[29] M. Tegmark and B. C. Bromley, ApJL 518, L69 (1999).

[30] M. Seaborne et al., MNRAS 309, 89 (1999).

[31] C. B. Netterfield et al., ApJ 445, L69 (1995).

[32] A. de Oliveira-Costa, A. Kogut, M. J. Devlin, C. B. Netterfield, L. A. Page, E. J. Wollack, ApJ 482, L17 (1997).

[33] G. F. Smoot et al., ApJ 396, L1 (1992).

[34] C. L. Bennett et al., ApJ 464, L1 (1996).

[35] M. Tegmark, D. J. Eisenstein, W. Hu, and A. de OliveiraCosta, ApJ 530, 133 (2000).

[36] A. de Oliveira-Costa, M. Tegmark, M. J. Devlin, L. M. Haffner Haffner, T. Herbig, A. D. Miller, L. A. Page, R. J. Reynolds, S. L. Tufte, ApJL 542, L5 (2000).

[37] B. C. Bromley and M. Tegmark, ApJL 524, L79 (1999). 